\title{
A Simplified Forest Fire Detection System and Improved Area Detection Method for Handling Exception
}

\author{
Seung-Hwan Yeom, Hye-Youn Lim and Dae-Seong Kang \\ Department of Electronic Engineering, Dong-A University New Media \\ Communication Lab. Busan Republic of Korea \\ aniz4you@gmail.com,ldalki07@hanmail.net,dskang@dau.ac.kr
}

\begin{abstract}
\end{abstract}
The purpose of this paper is real time forest fire detectibn system/ changing of the background and the foreground. This study is focused on easy case benreen Hardware and software, and made up widely used methods as a basis for each step First step is a process for detecting targeted forest fire by using HSV color'space. On second step, adaptive threshold used for more accurate and intuitive representation on forest fire area. And third step labeling used for distinguishing the targen area. Finalstep, histogram back-projection used for processing exception situation. By using proposed a method in this study, stable operation and more reliable method iscprove by simple experiments on detecting of the foreground.

Keywords: exception processing, Simple system, color space, histogram back-projection, forest fire

\section{Introduction}

Image processing in the current age is a technology which is used in all fields. It is also widely applied in facral detection and recognition tasks, improving the quality of color images and medical inages and enhancing document processing procedures, video summarizing, factory automation processes, content-based image searches, security issues, and disaster monitoring systems. The present study focuses on disaster monitoring systems.

Due to the charactefistics of Korean geography, with the many mountainous areas in the country, the distribution and ratio of forested areas are very high. Table 1 shows the five-year statistical data of forest area ratios for seven cities and nine provinces in Korea [1].

\section{Table 1. Statistical Forest Area Ratio Data for Five Years}

\begin{tabular}{|c|c|c|c|c|c|}
\hline $\begin{array}{l}\text { Total } \\
\text { district }\end{array}$ & $\begin{array}{l}2006 \\
\text { Year }\end{array}$ & $\begin{array}{l}2007 \\
\text { Year }\end{array}$ & $\begin{array}{l}2008 \\
\text { Year }\end{array}$ & $\begin{array}{l}2009 \\
\text { Year }\end{array}$ & $\begin{array}{l}2010 \\
\text { Year }\end{array}$ \\
\hline Total (\%) & 64.1 & 64.0 & 63.9 & 63.8 & 63.7 \\
\hline
\end{tabular}

As shown in the Table 1, forest areas are decreasing, but forests continue to cover more than the half of South Korea.

One instance of major damage caused by a forest fire was damaged in the amount of KRW 2.344 billion caused by a fire in Gangneung on March 29th 1998. Other examples are KRW 685 million in damage by a fire on the eastern coast on the same day, which also caused two deaths and 15 injuries; damage amounting to KRW 1.001 trillion by a fire on the eastern coast in 1999; and a total bill of KRW 23 billion by a fire in Yangyang in 2004. Thus, forest fires 
do not occur as often as general fires, but when they do occur, they are difficult to extinguish and typically incur high human and material costs [2]. In addition, several disaster detection systems are implemented, but each one is tailored to fit for constraints in the environment of a city or a non-residential (relatively few constraints of installing a sensing device in a small size and location), location and area (simplifying the size of the sensing device in mountainous areas), cost, etc.., also considered for efficiency.

Based on considerations, this system (software + hardware) aims to create for less expensive cost, adaptive to the mountainous area and to gain reliable sensing information from adaptively processing variables that cause for changing of the external environment (wind), first, the method of this study described explanation of the obtaining reliability by adding processes for software methods and exception conditions. This paper is organized as follows. In section 2, used theory is introduced. The proposed method for handling exception is described in section 3. The experimental results and processing speed are given in section 4 . Finally conclusions and the future research direction are discussed.

\section{Related Research}

\subsection{HSV Color Space}

Something that cannot be left out in image processing is known as the color space. The frequently used color spaces include the CMVK color space, the RGB color space, the CIE color space, and the HSV color space. Each of these offor spaces was developed and is used according to the service environment For example, the most frequently used color space, RGB, has equal values for each lighting component of $R, G$, and $B$. For this reason, it is not used in complicated backgrounds with extreme changes in lighting. From these color spaces, HSV is the most similar to the yisual system of humans; it is strong against light intensity and was formulated to proyidea more intuitive color designation method for the user. The HSV color model was used to process the golor space.

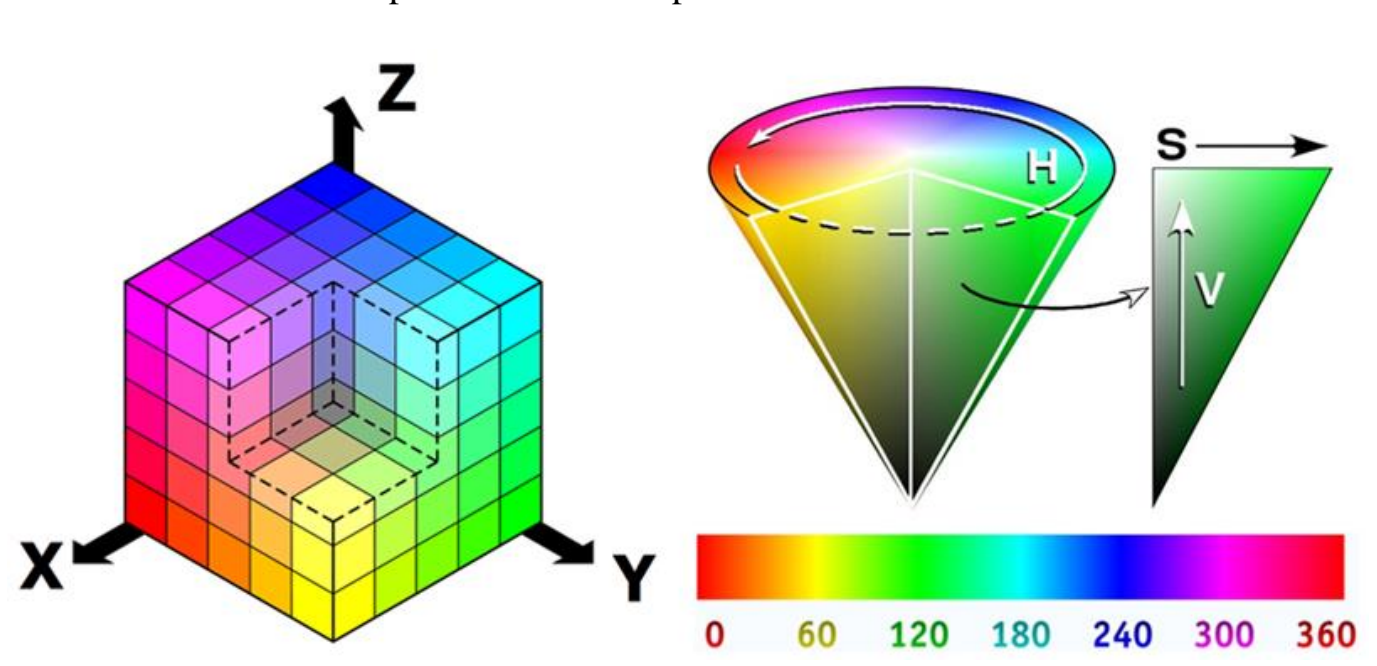

Figure 1. RGB and HSV Color Space Structure

Figure 1 in HSV, H stands for Hue, S for Saturation, and V is for Value. Hue is expressed as the angle of rotation around the vertical axis, with a value ranging from 1 to 360 . (Complementary colors are located at $180^{\circ}$ on the opposite side, with each becoming a complementary color when an angle of 180 is added.) Saturation has a value from 0 to 1 (in most software, it is expressed as $0 \sim 100 \%)$ and saturation becomes closer to $0(0 \%)$ as it 
becomes closer to the central axis and closer to $1(100 \%)$ as it moves away from the central axis. It has a value ranging from 0 to $1(0 \% \sim 100 \%)$ from the vertex of the cone to the uppermost layer. Therefore, when $\mathrm{V}=1(100 \%)$ and $\mathrm{S}=1(100 \%)$, each color becomes a pure color, while when $\mathrm{V}=1(100 \%)$ and $\mathrm{S}=0(0 \%)$, the color becomes white [3].

\subsection{Threshold and Adaptive Threshold}

When performing various image processing tasks, there are cases in which only pixels with a higher value than a threshold are left while the remaining pixels are ignored (though opposite cases exist). In these cases, a threshold is used to obtain results that are closer to the target.

The mechanism is as follows. The threshold value is pre-designated, and when the pixel values in the image are given, they are compared to the designated threshold values to perform the given operation. When this threshold value is used, the object image detected based on the color space can be shown more clearly and is thus easien to detect. However, threshold values are a result obtained after assigning a certain value to the image pixel irrespective of the external environment. Therefore, such a value has the disadvantage of being less adaptive. For this reason, threshold values are used. This method is an improved form of the existing threshold value method. The basic concept is identical to that of the threshold value method, but the distribution of the surrounding pixels is analyzed to designate the threshold value adaptively. Therefore, it is helpful when righting or light rapidly changes, is reflected, or is weak [4].

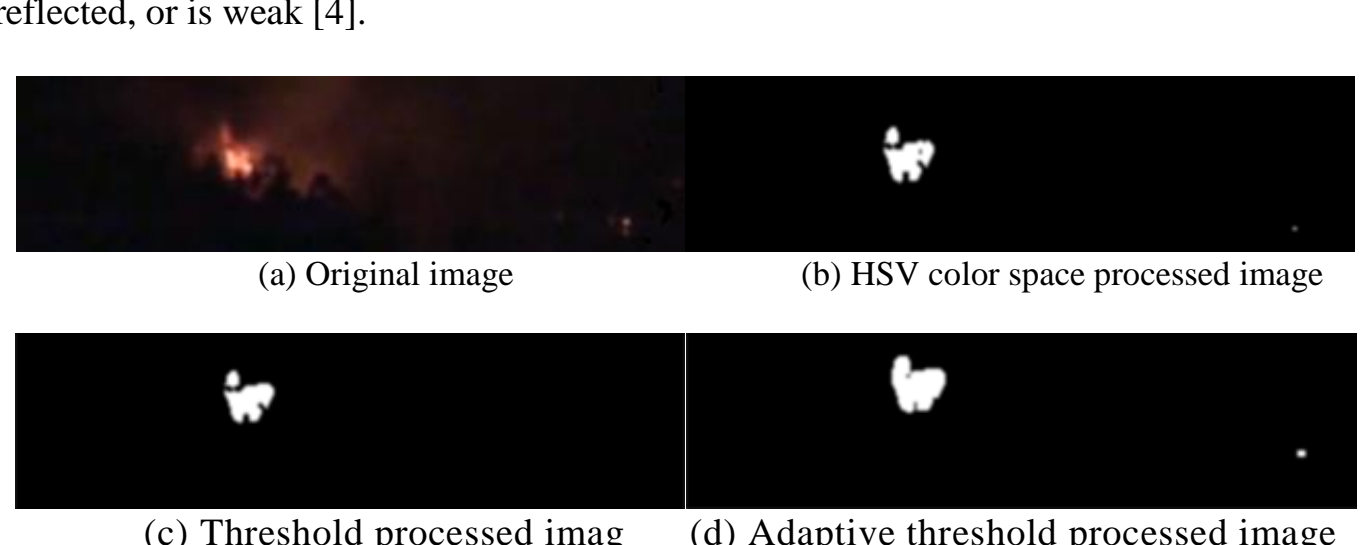

(c) Threshold processed imag

(d) Adaptive threshold processed image

\section{Fígure 2. Comparison of each Processed Image}

Figure 2 (a) shows the original image, and Figure 2 (b) shows the image after conversion of the HSV color space based on the original image. In addition, (c) and (d) show the threshold value and adaptive threshold value, respectively. The faint detected area in the bottom right area in (b) is not detected in (c) but is clearly detected in (d). Therefore, it is clear that an image processed with adaptive threshold values shows better results.

\subsection{Labeling}

'Labeling' simply refers to differentiating certain areas of pixels, which are the basic unit in an image. For example, in a forest fire image, if the area wanted by the user is the area where the fire exists or an area where there is smoke, this technique is used to detect or differentiate such areas. Figure 3 shows the most basic labeling process. In the traditional method, *symbol indicates areas that are not yet scanned. All of the colored space is the desired object detected using the HSV color space. Scanning moves from top to bottom and 
left to right, and two scanning methods are used. A four-neighbor connection assumes that only the pixels above, below, right, and left of a particular pixel are connected, while the eight-neighbor method assumes that even the pixels located diagonally are connected. This paper selected four-neighbor connection method for making the best use of Intuitive area.

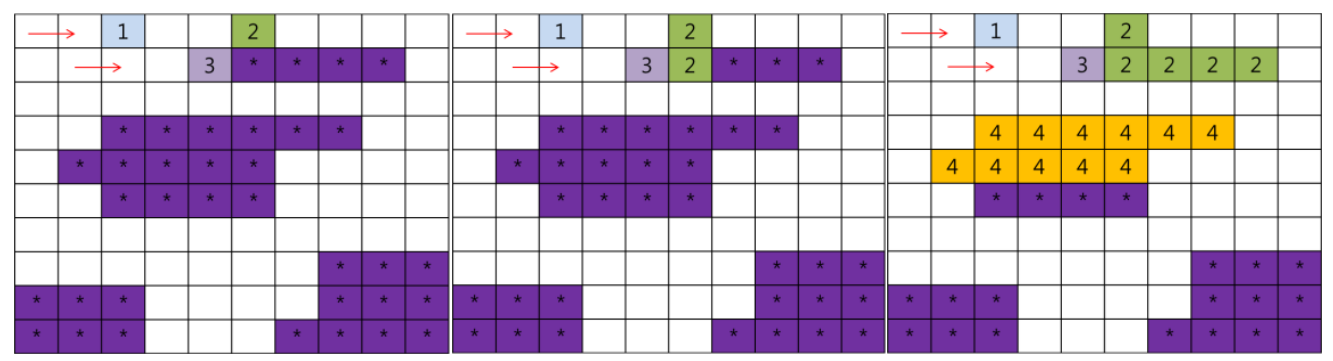

(a) Start

(b) Step 1

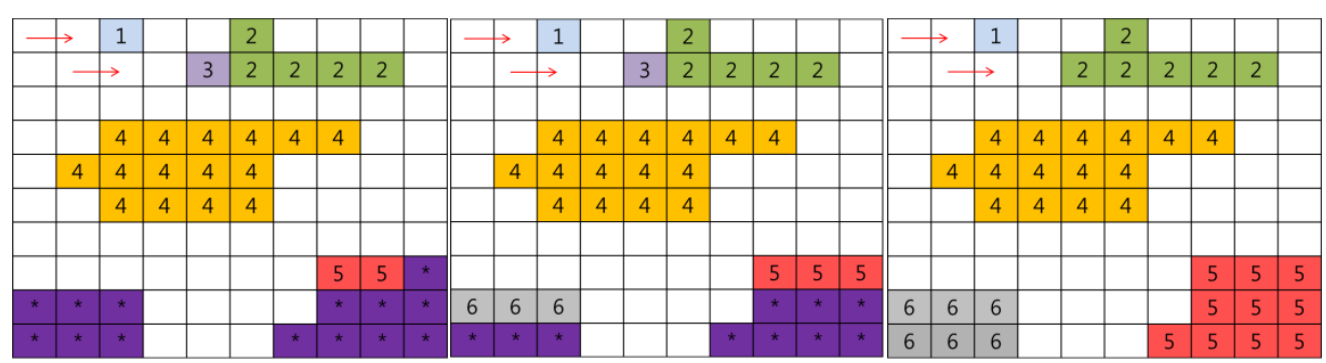

(d) Step 3

(e) Step 4

(f) Final result

Figure3. Materialization Stage of Labeling

Figure 3 (a) starts from the starting point and gives detected areas a number in order while scanning via the methodexplained above. When process 4 in Figure 3 (e) and the final results of Figure 3 (f) is examined, the pixels designated as number 2 and 3 respectively change to number 2 in the final results. This groups the vaguely detected number 3 with 2 to show more intuitive and accurate results.

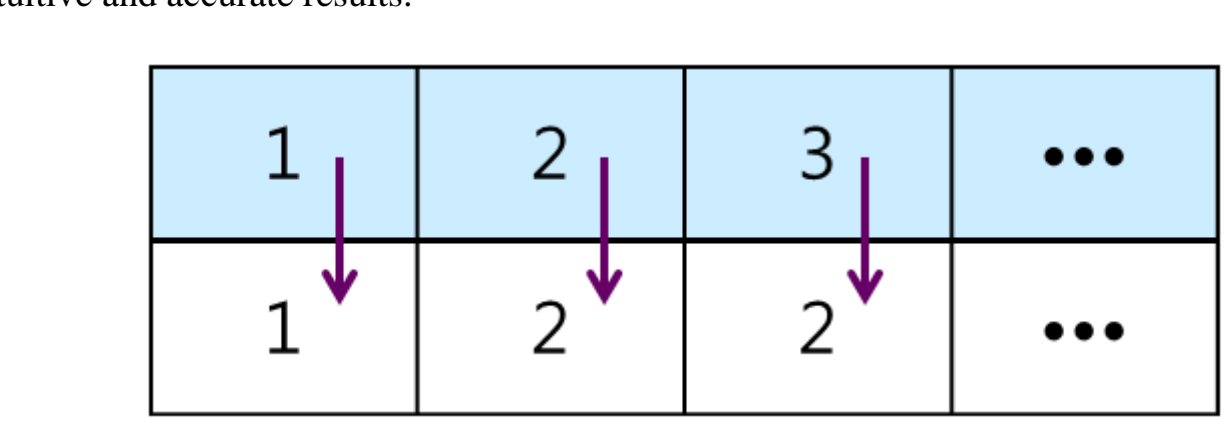

Figure 4. Method of Storing Labels in an Equivalent Table

The grouping method uses an equivalent table. If a random pixel in Figure 3 is defined as label 1, there are no other surrounding pixels to be defined according to the four-neighbor connecting method. Hence, it is defined as label 1 in the equivalent table, and this continues for label 2. Then, in the next line when label 3 is defined, label 2 is designated next to it. Thus, in the equivalent table, it designates the smaller value of label 2 from the labels neighboring label 3. Figure 4 shows how labels are designated in the equivalent table [5]. 


\subsection{Histogram Back-projection}

Most of situations don't maintain same situation, and irregular situations occur almost $100 \%$. Same situations occur on proposed system from this paper, and it is difficult to process exceptions [6]. From proposed system, irregular situation occurs on the Figure 5 - 318 frame.

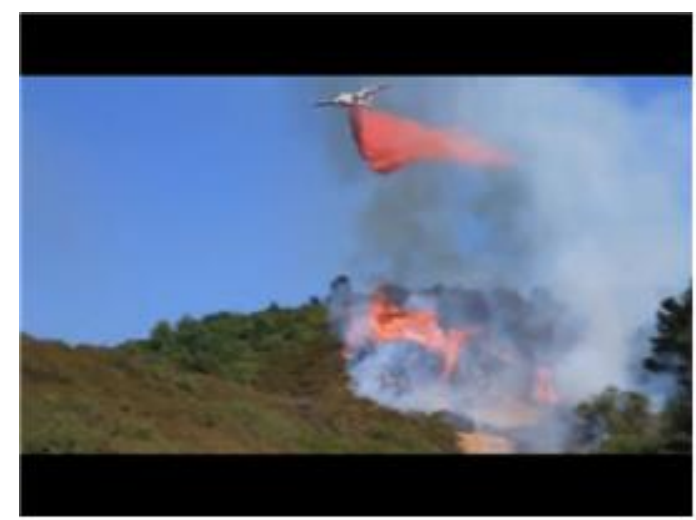

(a) Irregular situation frame (318)

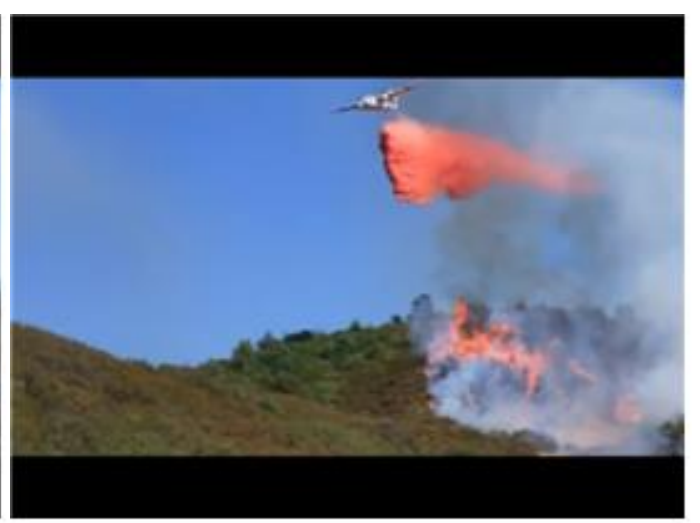

(b) Irregular situation frame (325)

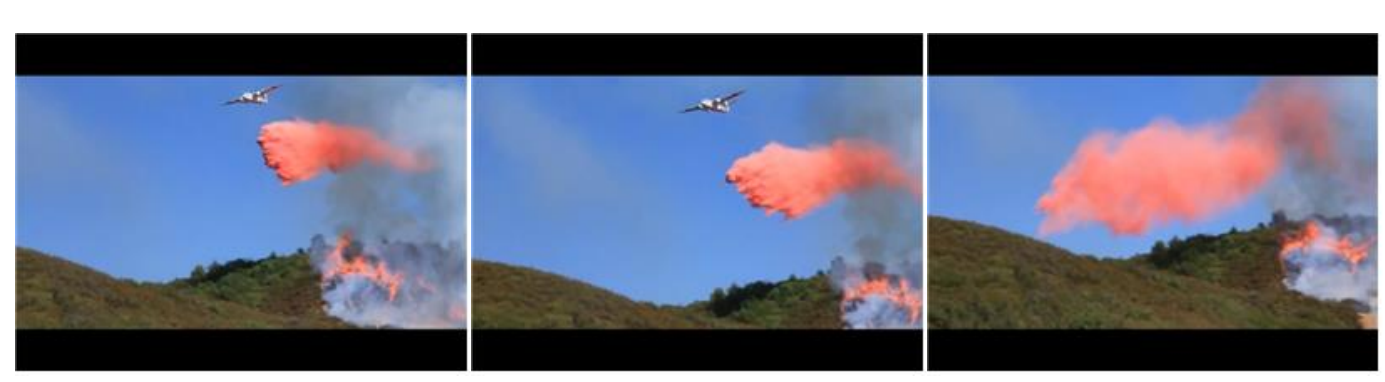

(c) Irregular situation frame $(332,340,392)$
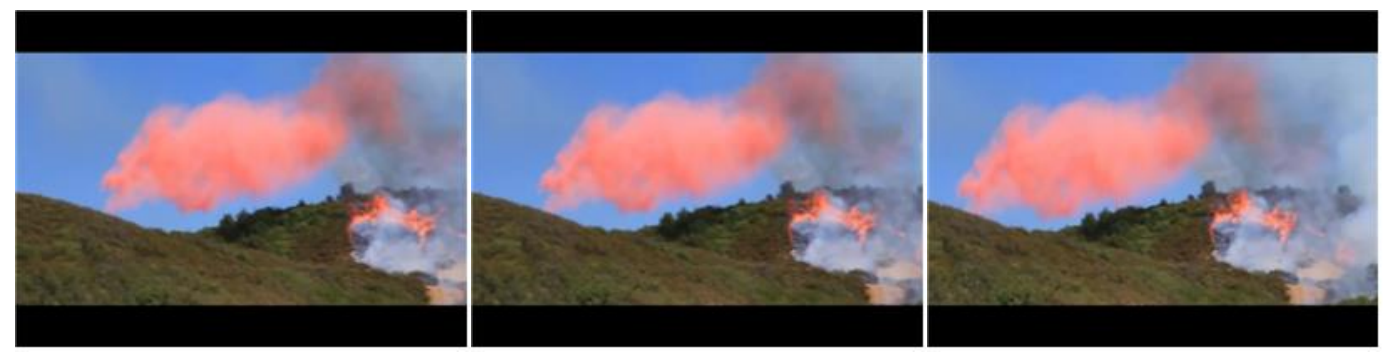

(d) Irregular situation frame (394, 396, 400)

\section{Figure 5. The Example Images of Exceptional Situation}

When plain sprays fire-extinguishing chemicals that have similar color with forest fire, system misrecognizes to forest fire like Figure 6. 


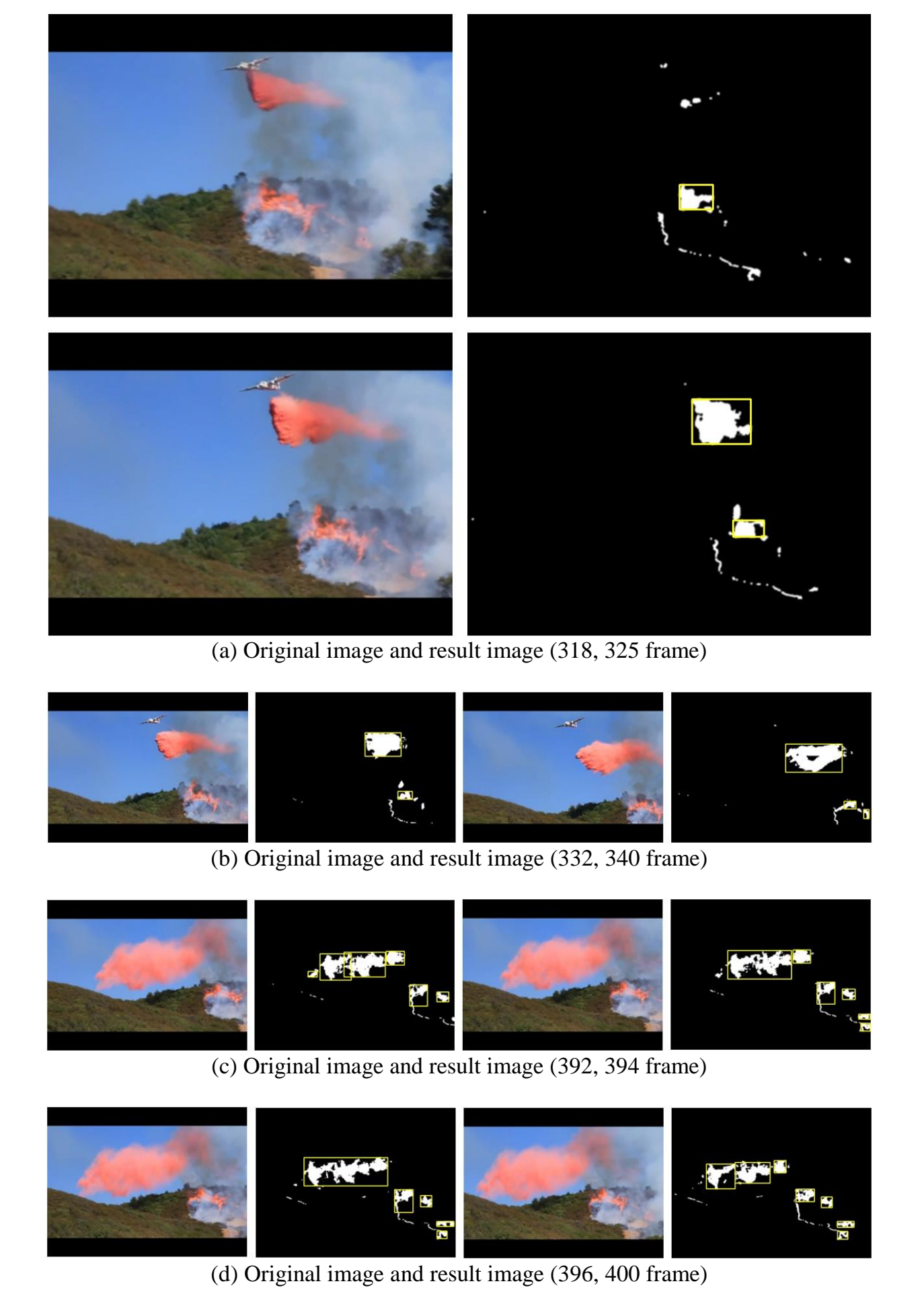

Figure 6. Exceptional Situation of Processing Result Image

Histogram back-projection used for processing irregular situations. And histogram backprojection needs the histogram information for original image, necessary object and area. Correlation, which basic method during a number of ways, used for comparing methods of two information [7-8]. 

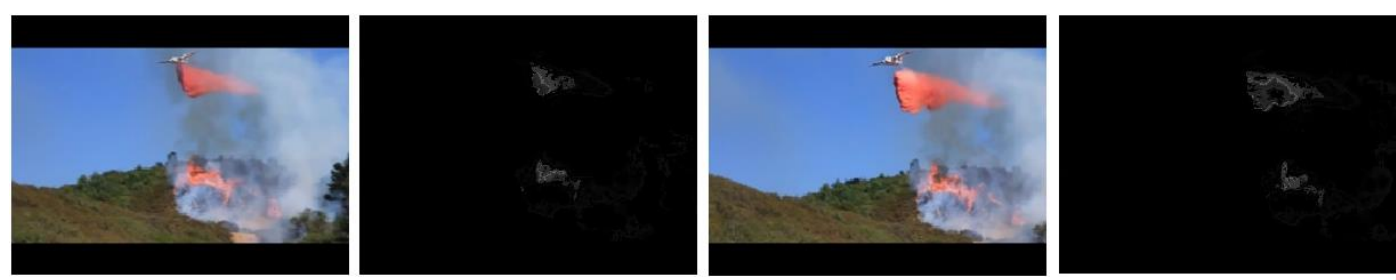

(a) Original image and histogram back-projection image (318, 325 frame)
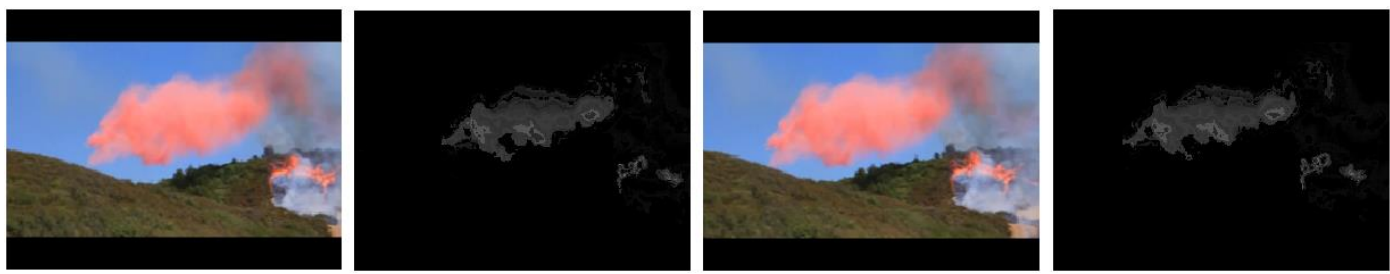

(b) Original image and histogram back-projection image (3,52,394 frame)

Figure 7. Back-projection Images Using Histogram in Exceptional Situation
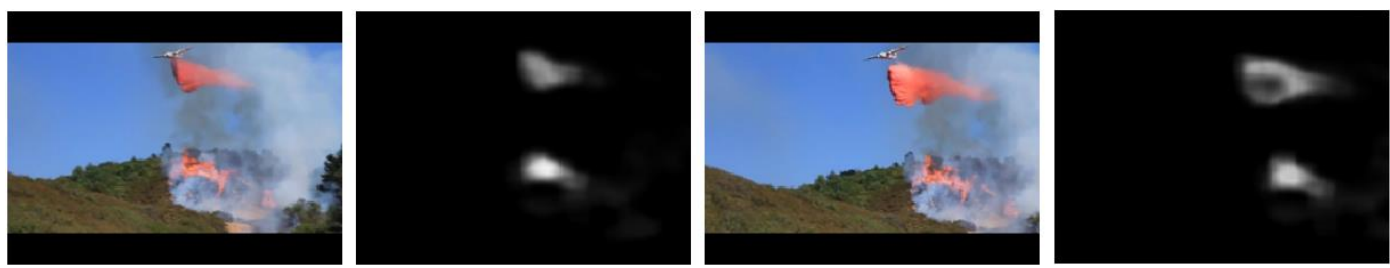

(a) Original image and patch bistogram back-projection image (318, 325 frame)
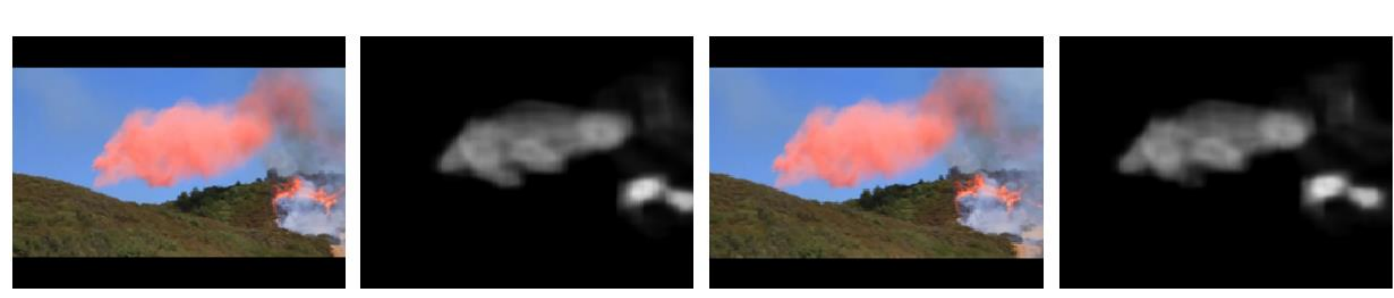

(b) Origina image and patch histogram back-projection image (392, 394 frame)

\section{Figure 8. Back-projection Images Using Patch Histogram (the brightest area = detection area)}

On Figure 7, histogram back-projection is no special function. But when histogram backprojection method uses patch, it shows better advantage like Figure 8 than Figure 7. This method is, after analyze the wanted area (object) from pre-treated in the interest area (labeling), it is method to recognize highest similar area from the hole area. This is method use all pixels from wanted area, not one pixel. It recognizes characteristic (color information, object location, probability distribution, etc.) of the histogram and also texture, etc. so these information use for detecting more accurate area and object [9-11]. 


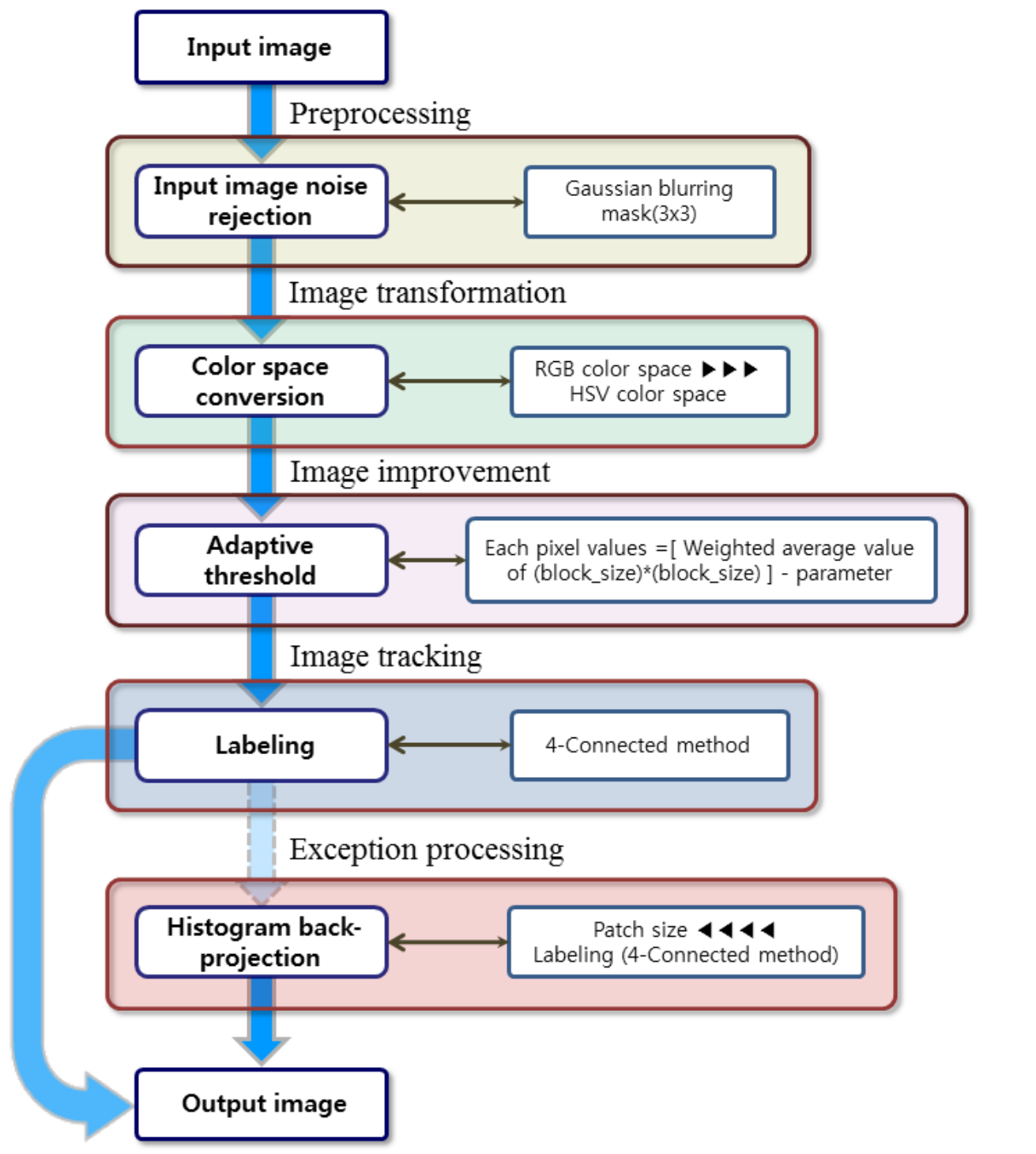

Figure 9. Block Diagram of the Proposed Method

\section{Proposed Method}

\subsection{Environment}

As mentioned in the introduction, in fact, considering the incidence of fire and monitoring range, former disaster prevention system focus on highly cost-effective for forest fire detection system. Unlike center of a city, several environmental variables exist in mountainous area. So the forest fire detection system needs low-cost, high-efficiency and accurate tracking system (required needs, simple installation and distinct detection ability even under exceptional situations).

The target in an environment that has a lot of variation, also installation should be simple. Urban areas are easy to monitor and maintain. But mountainous terrain is hard to granular of the monitored area and the install limitation on size of monitoring device. Because mountainous terrain is much too wide. In this paper the examples screen flicker and exception processing by winds among several environmental factors [12]. 


\subsection{Proposed System}

To realize a simple (high expandability in terms of hardware, but with less processing required by the software) and the adaptive system, the constructed algorithm was intended to be very simple. To achieve the desired purpose, the method shown in Figure 9 is suggested. First, when the image is inputted, Gaussian blurring is performed as a preprocessing step to minimize noise. Then, through an image transformation process, the basic color space of RGB is converted to HSV, with image improvement subsequently performed with adaptive threshold values to heighten the degree of adaptation to the intensity and reflection of the lighting or light. Finally, after implementing final results by using labeling for detecting necessary area, histogram back- projection used for processing the exceptional area.

\section{Realization}
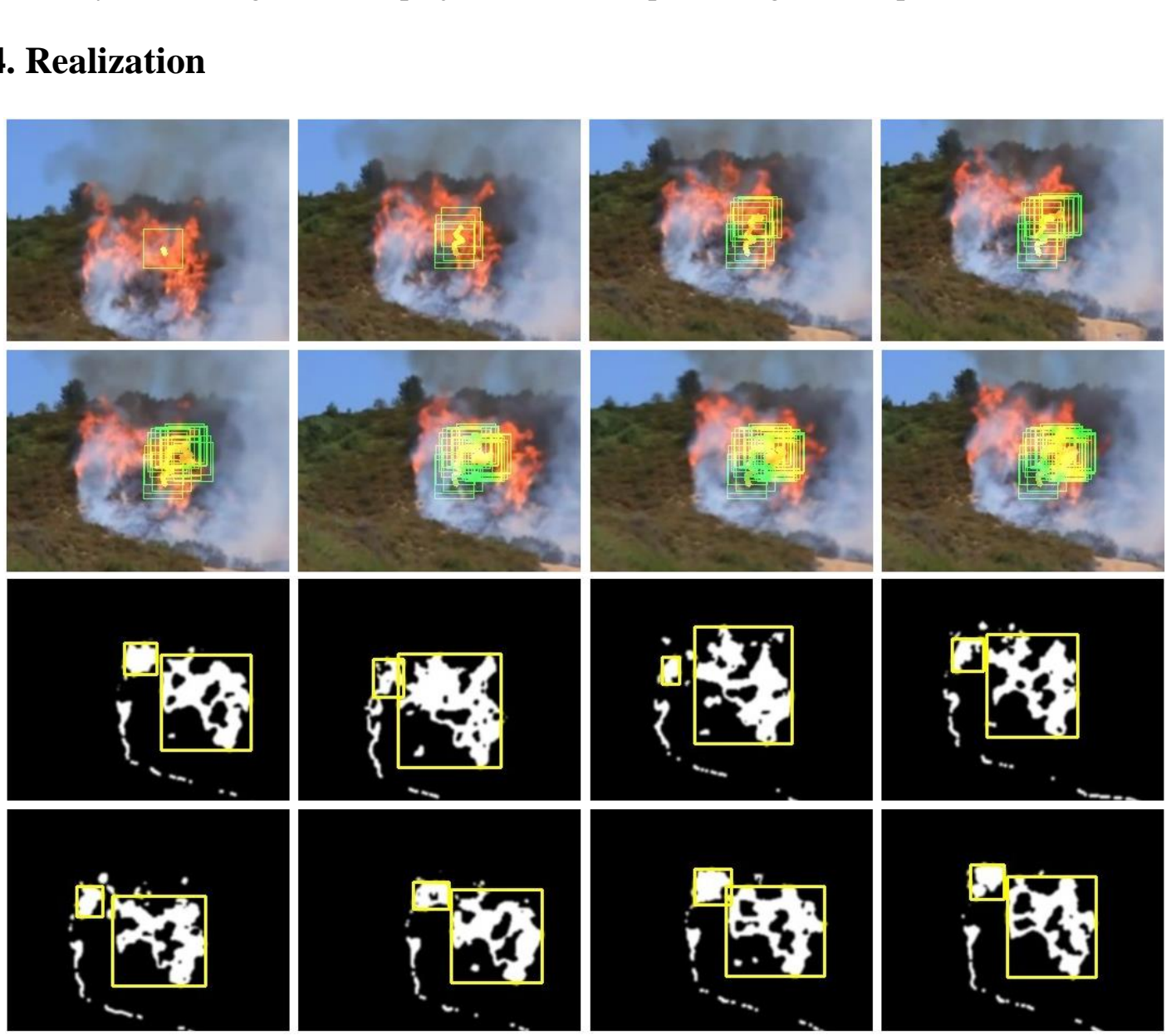

Figure 10. When the Whole View and Background are Moving Together (shaking of camera, 5 frame intervals)

To aiming at adaptability (Camera (screen) shake, exception situation), this paper was used more shade video than other video. 

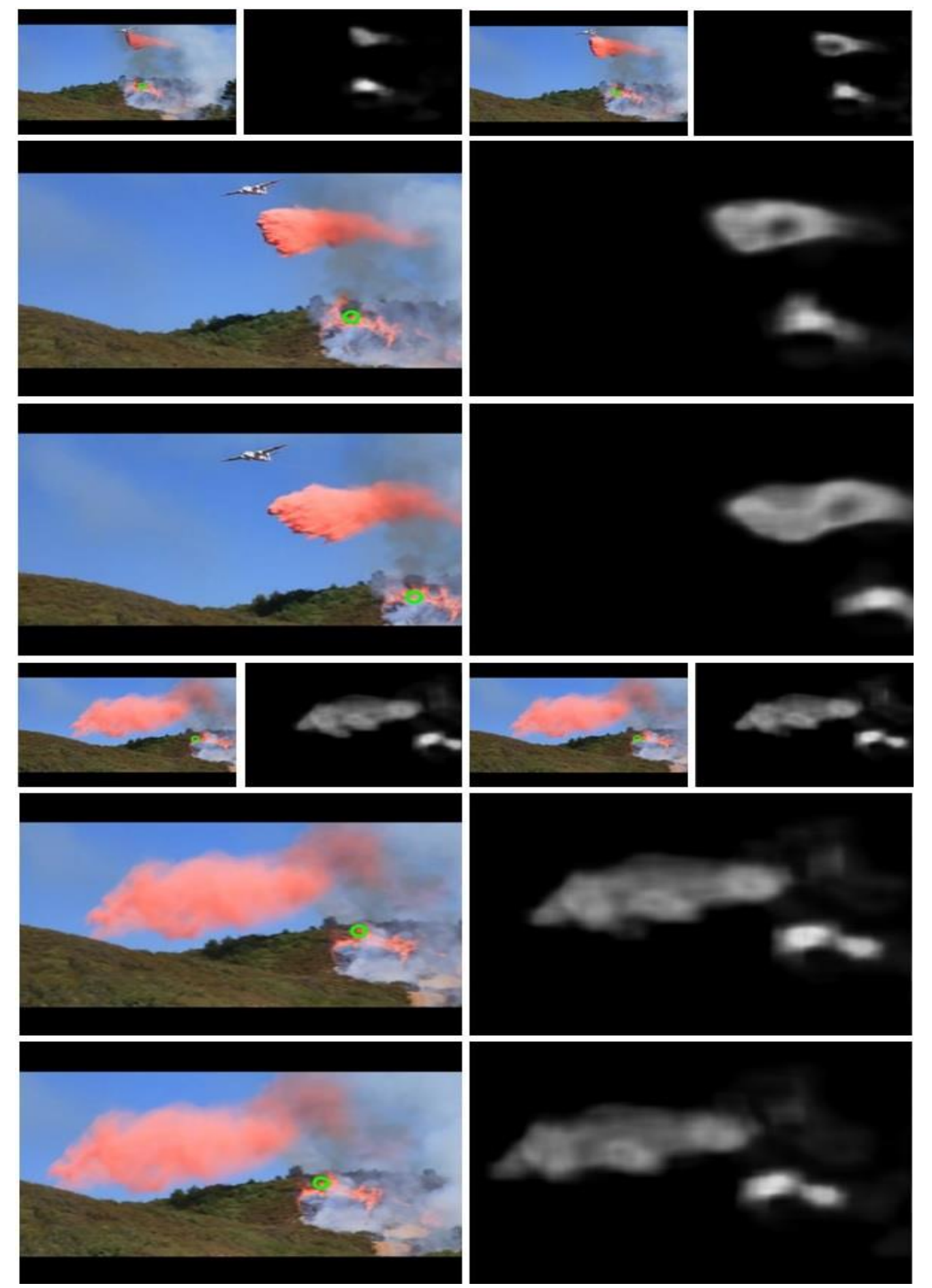

Figure 11. The Resulting Images Using the Proposed System (318 400 frame, green circle $=$ detection area)

Table 2. Performance Evaluation of Proposed System (processing speed)

\begin{tabular}{|l|l|l||l|l|} 
Time / sec & Average processing time & Frame & Frame & Processing speed / \\
\hline
\end{tabular}




\begin{tabular}{|c|c|c||c|c|}
\hline & / sec & rate & & sec \\
\hline $0 \sim 1$ & 0.028078 & $24 \mathrm{fps}$ & 1 & 0.032604 \\
\hline $1 \sim 2$ & 0.028075 & $24 \mathrm{fps}$ & 2 & 0.042849 \\
\hline $2 \sim 3$ & 0.028082 & $24 \mathrm{fps}$ & 3 & 0.028127 \\
\hline $3 \sim 4$ & 0.028086 & $24 \mathrm{fps}$ & 4 & 0.028642 \\
\hline $4 \sim 5$ & 0.028076 & $24 \mathrm{fps}$ & 5 & 0.028372 \\
\hline $5 \sim 6$ & 0.028085 & $24 \mathrm{fps}$ & 6 & 0.028220 \\
\hline $6 \sim 7$ & 0.028099 & $24 \mathrm{fps}$ & $\vdots$ & $\vdots$ \\
\hline $7 \sim 8$ & 0.028108 & $24 \mathrm{fps}$ & 284 & 0.028889 \\
\hline $8 \sim 9$ & 0.028101 & $24 \mathrm{fps}$ & 285 & 0.028851 \\
\hline $9 \sim 10$ & 0.028115 & $24 \mathrm{fps}$ & 286 & 0.028923 \\
\hline $10 \sim 11$ & 0.028128 & $24 \mathrm{fps}$ & 287 & 0029367 \\
\hline $11 \sim 12$ & 0.028123 & $24 \mathrm{fps}$ & 288 & 0.028962 \\
\hline $\begin{array}{c}\text { Total average } \\
\text { processing time }\end{array}$ & 0.028096 & & $\begin{array}{c}\text { Total average } \\
\text { processing time }\end{array}$ & 0.030106 \\
\hline
\end{tabular}

Table 3. In Exceptional Situation, Processing Speed of Each Frame (eight frames)

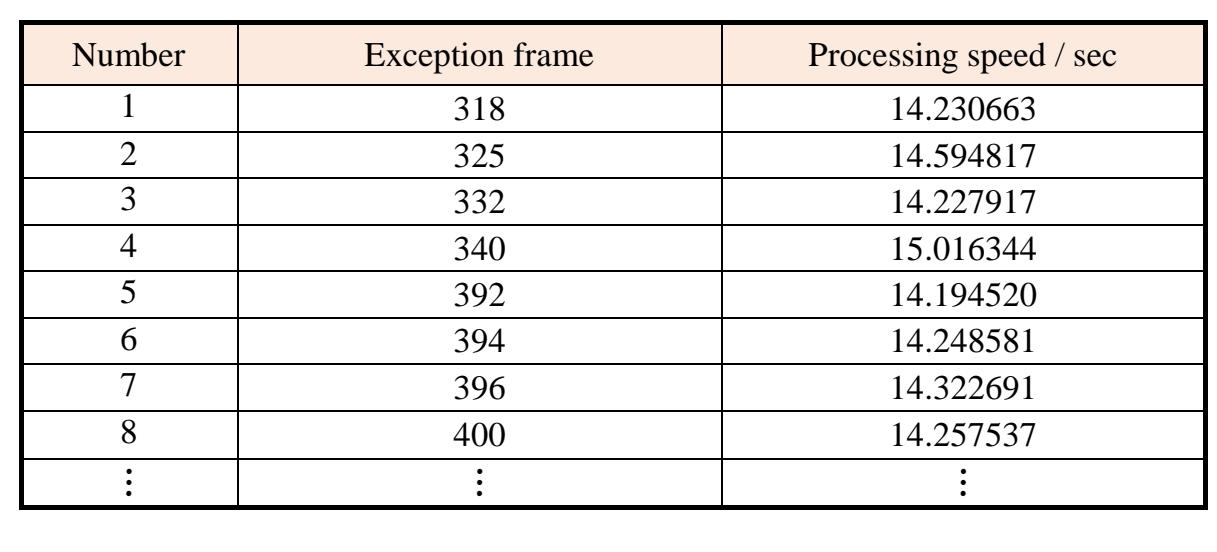

\section{Conclusion and Direetions for Further Research}

Figure 10 and Figure 11 show, it is detected reliably on determined area. Table 2, so simple hardware was comprised of average of the processing speed in 12 seconds based on 24 frames per second ard the average about 0.03 , the processing speed on each frame. And it is possible to process in real-time.

The experimental results show the used image had based daytime that effect on brightness, also hard to recognize, the video had shaking image, the background and foreground blur rapidly changed (see Figure 10/ Top side) [13].

Target area was Fast and reliably detected from shaking image base on color space without difference image technique (unusable image to rapidly change in each frame of image), one of the basis object detection methods. Like Figure 11 (upper left), first irregular situation frame from patch of forest fire use for gaining reliable result by processing other irregular situation frames. And also, when irregular situations occur from 318 400 frame in Figure 11, wanted area reliably detected on frame. The reason of this result is utilization of forest fire texture information, like the probability information or color information.

However like Table 3, method has disadvantage that can't apply real-time cause of high computation instead have high reliability. The most needed improve point is Modified and supplemented for real-time in this system. Based (one chip processor) on micro controller, 
hardware operates without problems. Additional elements (detection of other targeted area, etc.,) are implemented besides forest fire detection system by later study.

\section{Acknowledgements}

This research was supported by Basic Science Research Program through the National Research Foundation of Korea (NRF) funded by the Ministry of Education, Science and Technology (2011-0011735).

\section{References}

[1] www.forest.go.kr / Statistical forest area ratio data.

[2] www.nema.go.kr / Fire Prevention Key Statistics 2012.

[3] L. Shuhua and G. Gaizhi, "The application of improved HSV color spage model in image processing", Future Computer and Communication (ICFCC), 2010 2nd International Conference on vol 2)(2010), pp. V2-10-V213, May.

[4] X. Deng, Y. Huang and S. Feng, "Adaptive threshold discriminating algorithm for remote sensing image corner detection”, Image and Signal Processing (CISP), 20103 rd International Congress on v.2, (2010), October, pp. $880-883$.

[5] G. Zankl, Y. Haxhimusa and A. Ion, "Interactive Labeling of Image Segmentation Hierarchies", Joint 34th DAGM and 36th OAGM Symposium 2012, (2012), pp. 11 - 20, August.

[6] C. Fetzer, P. Felber and K. Hogstedt, "Automatic Detection and Masking of Nonatomic Exception Handling", IEEE Transactions on Software Engineering,

[7] G. Bradski and A. Kaehler, "Learning OpenC Keomputer Vision with the OpenCV Library", Publisher O'Reilly Media, Released: (2009) September.

[8] J.-h. Lee, W.-h. Lee and D.-s. Jeong, 'Object tracking method using back-projection of multiple color histogram models", Circuits and Systems, 2003。 ISEAS '03, Proceedings of the 2003 International Symposium on vol. 2, (2003) May, pp. 668 - 671.

[9] X. Chen, Q. Huang, P. Hu, M. Li.) Y. Tian and C.L1, "Rapid and Precise Object Detection based on Color Histograms and Adaptive Bandwidth Mean Shite", The 2009 IEEE/RSJ International Conference on Intelligent Robots and Systens, (2009) Qctober 11-15, St. Louis, USA.

[10] I. R. Khan and F. Farbiz, "A Back Projection Scheme for Accurate Mean Shift Based Tracking”, Proceedings of 2010 IEEE 17th Hnternational Confe-rende on Image Processing, (2010) September 26-29, Hong Kong.

[11] J. G. Avina-Cervantes, S. Ledezma-Orozco, M. Torres-Cisneros, D. Hernandez-Fusilier, J. Gonzalez-Barbosa and A. Salazar-Garibay, "Cold-texture histograms for natural images interpretation", Sixth Mexican International Conference on Artificial Intelligence, Special Session, (2007) Nov 4-10, Aguascallentes, Mexico.

[12] H.-Y. Lim and D.-S.Kang "A Study of Advanced Tracking Algorithm using Color Probability Distribution for Objects Tracking System”, Journal of Korean Institute of Information Technology, vol.8, no.2, (2010), pp. 153-158.

[13] H. S. Jeon, D -H.YYeom and Y. H. Joo "Video-based Intelligent Unmanned Fire Surveillance System", Journal of Korea Institute of Intelligent Systems, vol. 20, no.4, (2010), pp. 516-512.

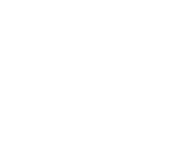




\section{Authors}

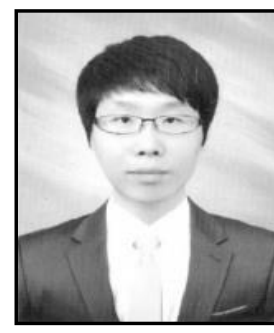

Seung-Hwan Yeom, he received his B.S. degree in Electronics Engineering from Dong-A University, Korea, in 2013. His research interests include computer vision, object detection and tracking.

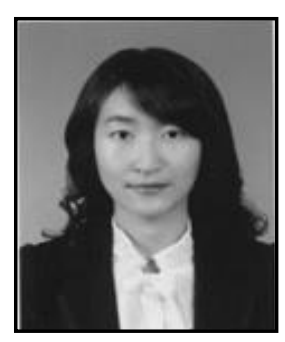

Hye-Youn Lim, she received his B.S. degree from Daegu Oniversity, Daegu, Korea, in 2005 and his M.S. and Ph.D. degrees in Electronics Engineering from Dong-A University Bussan, în 2010 and 2013, respectively. She has been an assistant professor in Department of Electronics Engineering, Dong-A University. He research interests include image processing and object tracking.

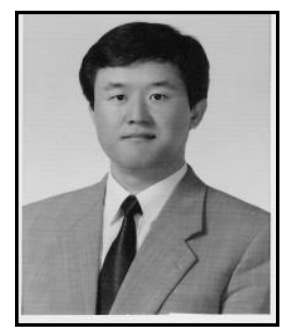

Dae-Seong Kang, he reeeived his B.S. degree from Kyungpook national Unifersity,' Daegu,KKorea, in 1984 and his M.S. and Ph.D. degrees in Electronics Engineering from Texas A\&M University, USA, in 1991 and 1994, respectively. He has been a professor in Department of Electronics Engineating, Dong-A University. His research interests inçlude umage processing and compression.

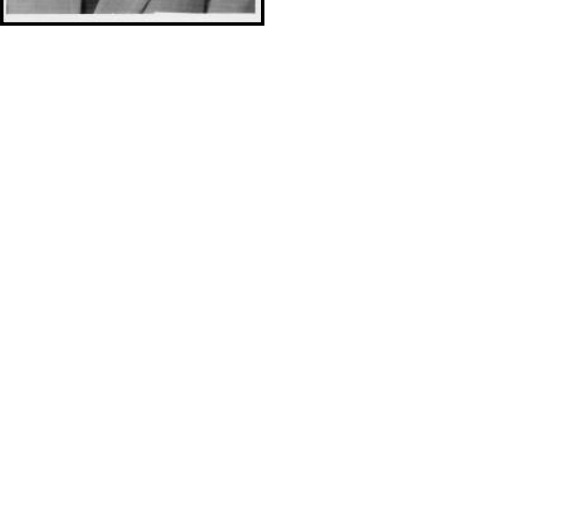


International Journal of Multimedia and Ubiquitous Engineering Vol.9, No.7 (2014)

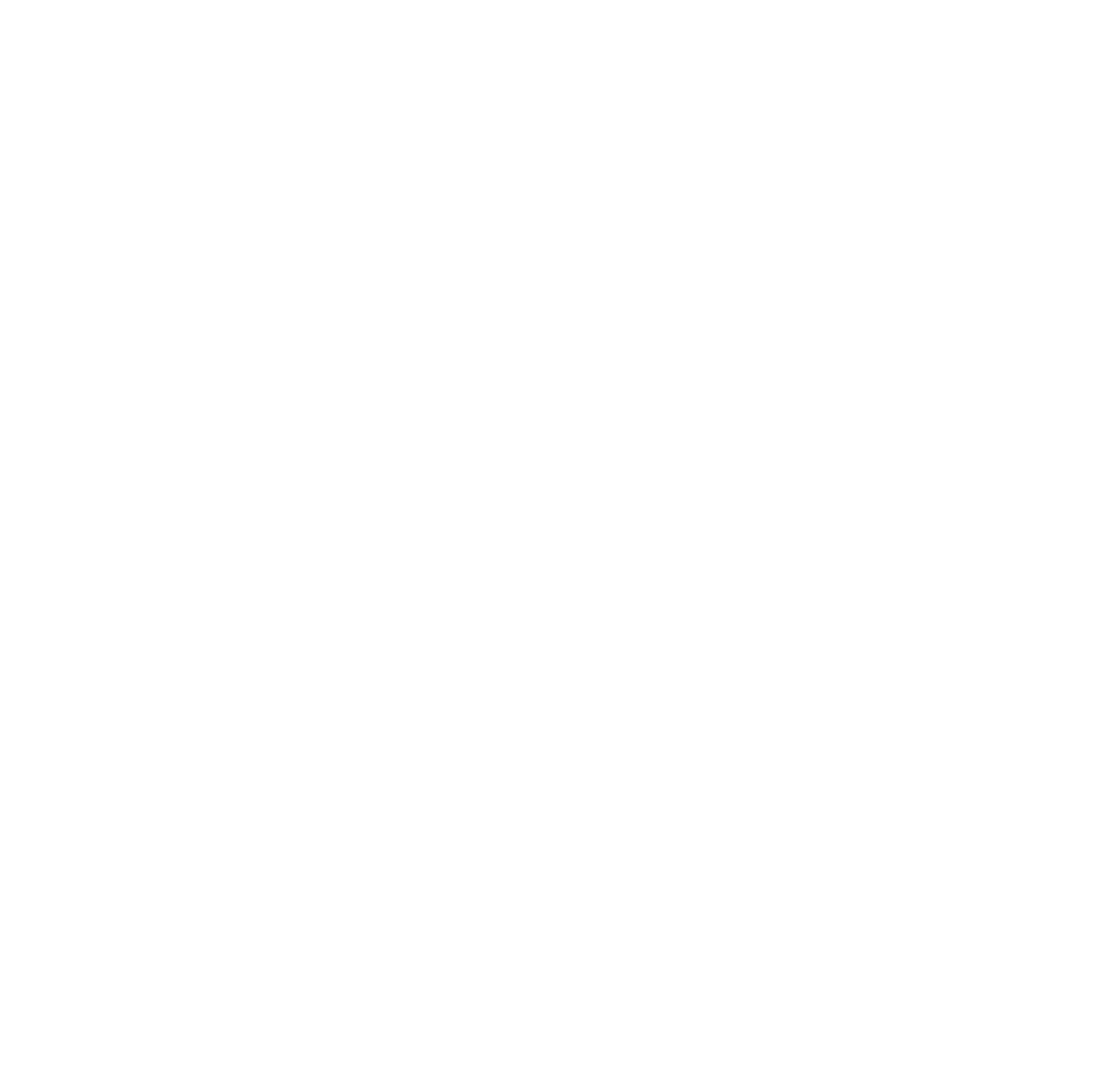

